\title{
Supported Decision-Making: Non-Domination Rather Than Mental Prosthesis
}

\author{
By Allison McCarthy and Dana Howard \\ Forthcoming in AJOB Neuroscience
}

\begin{abstract}
Recently, bioethicists and the UNCRPD have advocated for supported medical decision-making on behalf of patients with intellectual disabilities. But what does supported decision-making really entail? One compelling framework is Anita Silvers and Leslie Francis' mental prosthesis account, which envisions supported decision-making as a process in which trustees act as mere appendages for the patient's will; the trustee provides the cognitive tools the patient requires to realize her conception of her own good. We argue that supported decision-making would be better understood as a collaborative process, giving patients with intellectual disabilities the opportunity to make decisions in a respectful relationship with trusted others. We offer an alternative account of supported decision-making where the primary constraint is to protect the patient from domination by the trustee. This is advantageous in its preservation of the prospects for genuine collaboration, for the mental prosthesis approach ultimately reinforces a problematic ideal of isolated patient self-determination.
\end{abstract}

Keywords: decision-making, disability, autonomy, non-domination

\section{Introduction}

Anita Silvers and Leslie Francis have long argued that a liberal society should be inclusive not only in regard to people's unconventional values or views, but also in regards to the unconventional "cognitive processes whereby these conceptions are formulated and maintained." (Silvers and Francis 2009, 476) They paint a picture of what this radical inclusion would look like: while we should respect the evaluative perspectives of those people who can be characterized as "cerebral" and who actively engage in formulating, revising, and expressing their conception of the good, we must also respect those persons who "are so unable to express themselves in any way that we cannot be sure whether their behavior is mediated by concepts at all." (Silvers and Francis 2009, 476) This paper, in accordance with Silvers and Francis, starts from the assumption that people with intellectual and developmental disabilities, including those who are not verbally communicative, are owed respect as persons and asks how should decisions be made on their behalf. 
Consider the following case of a mother, Kristina Chew, who talks about the difficulties she faces when tasked with the work of representing her son, Charlie, who is autistic and minimally verbal:

The quandary in representing Charlie is that he, the one being represented, is, all too often, limited in his ability to speak back and up about himself. I write about my son knowing that I may be very wrong about what he experiences, that it may be a very long time before he uses language to tell me and that indeed that may never happen...the communications of "nonverbal" or "minimally verbal" autistic people - including Charlie — are routinely perceived as so difficult to decode that they verge on the untranslatable and unknowable. This perception invites us to displace autistic voices with our own.(Chew 2013, 305-6)

Three features are important to highlight about the way that Chew describes her predicament:

[1] There is always going to be a chance that Chew misunderstands Charlie's evaluative perspective or even whether he intends to communicate his evaluative perspective about a certain matter in a given instance with certain gestures or utterances.

[2] It is unclear whether Charlie chooses to or can offer her feedback on the ways in which she is interpreting and representing his evaluative perspective.

[3] The danger that Chew worries most about is that this communicative crevasse tempts her to supplant Charlie's evaluative perspective with her own.

Given these difficulties and the danger of obscuring the evaluative perspective of people like Charlie, how should we engage in decision-making that is radically inclusive of all of us, including those members of the human community who have intellectual disabilities?

There seem to be three options for decision-making regarding persons with profound intellectual and developmental disabilities. We could maintain that medical decisions on behalf of intellectual and developmental disabilities (hereafter referred to as 'IDD') patients should always be independently controlled by the patients themselves. This means that the patient retains unconditional authority to make their own medical decisions, including the decision to solicit the support or advice from third parties as they see fit. While this is an important live option for many 
IDD patients, it is unclear how such a deliberative process would be feasible for a person like Charlie who has minimal communicative capacities. As Chew makes clear, all interactions with Charlie, at least at his current developmental state, require some interpretive work and representation on the part of his interlocutors.

Second, we could institute a policy of substituted judgment. On this option, the trustee imagines what the patient would have chosen with access to full information and the ability to process it. Any relevant direct information for the substituted judgment standard is that which may have been expressed by the patient at a prior time of decision-making capacity. ${ }^{1}$ If no such information is available, this is not seen as an impediment to the deployment of the substituted judgment standard. In fact, the substituted judgment standard often is "speculative and reconstructive", to use Buchanan and Brock's language (Buchanan and Brock 1990, 119). Consequently, substituted judgment does not require - and often actively discourages - the solicitation on the part of the trustee for any actual patient input at the time of decision-making, it is enough for the trustee to decide on the basis of this hypothetical evaluative perspective of the patient were they to be fully informed. It is unclear how this method would respect Charlie as a person: why think his current preferences and values would match the preferences and values of a person with full information or with wholly different set of cognitive capacities? (Howard and Wendler 2020)

Finally, we can delineate a process of supported decision-making which envisions a collaboration between the patient and the trustee, but which gives the patient some sort of central authoritative role in the deliberation. Supported decision-making, at its heart requires two people

\footnotetext{
${ }^{1}$ It should be noted that trustees for patients who have previously been capable of medical decision-making (where the substituted judgment standard is most frequently invoked) are often instructed to disregard the input of a currently incapable patient, in favor of the trustee's imaginative reconstruction of the patient's evaluative perspective and the way the current decision at hand might fit in with this perspective.
} 
communicating together to reach a single decision. This can be contrasted with the single decisionmaker envisioned by either the independent control model or the substituted judgment model. On both of the first two models, there is a single decision-maker that is subject to certain constraints on their authority. For the independent control model, the patient retains decisional authority as long as they meet a certain threshold of cognitive capacity; if they fall below the threshold, they lose the authoritative standing of a person's whose values should be respected and come to be understood solely in their role as a patient with interests to be safeguarded. For the substituted judgment model, on the other hand, the authoritative standing of the trustee is determined by their good faith effort to approximate the patient's hypothetical independent evaluative perspective.

So on neither the independent control or the substituted judgment model is any actual interpersonal co-deliberation required for decision-making: either the patients themselves or their surrogate decision-makers have sole authority to make a decision. This is not the case with supported decision-making which constitutively requires actual exchange -- or at least the opportunity to do so -- between interlocutors.

Because of this distinction, supported decision-making has gained traction as the best model for medical decision-making for IDD patients who are designated as decisionally incapacitated. We agree that supported decision-making is the most promising path forward, but it is not one without its own share of challenges. Given the communicative barriers and the secondguessing that is pervasive in trustee-patient relations, how are we supposed to evaluate whether the supported decision-making has succeeded and whether the patient has been afforded the respect they are due? This paper suggests that what matters in a process of supported decision-making for IDD patients is that the patient's perspective on the good is afforded the same moral significance as the perspective of a patient who is capable of unsupported decision-making. We will argue that 
for both supported and unsupported decision-making, patients are respected when they are not dominated by another in their decision-making.

\section{Unacceptable Decision-Making for IDD Patients}

In considering how medical decisions ought to be made on behalf of IDD patients, it may be helpful to examine a case that illustrates, from our perspective, an unacceptable approach to medical decision-making for this patient population. In Doe ex rel. Tarlow v. District of Columbia (489 F.3d 376 (D.C. Cir. 2007)), the majority opinion (authored by then-Circuit Judge Brett Kavanaugh) states that in cases of IDD patients, decisions ought to be made on the basis of "a good faith belief as to the best interests of the patient", not "the known wishes standard" (Doe). Because these are patients who never have had the capacities required by law to engage in unsupported decision-making, "their true wishes with respect to a recommended surgery 'are unknown and cannot be ascertained'..." (Doe). That the plaintiffs in fact possessed and articulated clear wishes with respect to a recommended surgery was purportedly irrelevant to how decisions regarding their treatment should be made.

Doe reflects a specific way in which IDD patients are subject to disrespect in medical decision-making. Per Doe, because these patients lack, and have always lacked, certain capacities that are deemed necessary for making decisions by way of a purely rational process, it is impossible for these patients to have values or preferences or commitments that could be relevant to medical decision-making. The exact kinds of considerations that possess significant authority in decisionmaking for currently competent or "once-competent" are devoid of any weight in decision-making for "never-competent" patients. All decisions, according to Doe, should be made on the basis of the best possible outside judgment of what is in the patient's best interests. Consequently, not only is there no obligation for those tasked with making decisions on behalf of IDD patients to take 
their stated wishes and preferences into account; decision-makers, in many cases, should actively disregard patients' stated wishes and preferences, as they may lead to distorted, incorrect, or even harmful decisions for the patient.

In the clarity of its illustration of how IDD patients are denied recognition of their agential capacities, Doe helps us understand how medical decision-making for this patient population ought to be approached. Even if IDD patients may lack certain capacities (or fail to possess them to some specific degree) deemed necessary to make authoritative decisions without assistance, this should have no bearing on whether their actual wishes inform medical decisions. An approach to medical decision-making that treats the actual wishes of IDD patients as irrelevant, or even a potential hindrance, fails to give moral weight to these patients' evaluative perspective and is thus disrespectful (Howard and Wendler 2020).

Supported decision-making is particularly promising, then, because of the pride of place it gives to the actually elicited perspective of the patient. ${ }^{2}$ The approach is a direct response to the unique form of disrespect towards patients exemplified in Doe and endemic to current practices of medical decision-making. By treating as non-negotiable the relevance of the patient's perspective to the decision and committing to recognition of the patient as the source of the decision, supported decision-making centers the patient as an agent in medical decisions - even if that agency requires support to be enacted. In articulating the nature of the collaboration constitutive of supported decision-making, we propose that this is the paramount ethical concern.

\section{A Non-Domination Approach to Supported Decision-Making}

\footnotetext{
${ }^{2}$ Our discussion is applicable primarily to those IDD patients who are able to engage in kinds of activity that give clear indication of an evaluative perspective. Precisely what forms of activity can provide the basis for judgments about a patient's evaluative perspective is an important question that any practicable account of supported decisionmaking will need to address. But it is crucial, from our perspective, that we not assume that the kinds of activity able to form the basis of appropriate judgments about the patient's evaluative perspective are those the majority of people will comfortably recognize as communicative activity. Thank you to an anonymous reviewer for pressing us to clarify this.
} 
In the literature on medical decision-making for patients deemed capable of unassisted, authoritative decision-making, it is common to discuss the patient's values as a constraint on and a guide for medical decisions. But 'values' often picks out a set of attitudes that are quite cognitively sophisticated; they are something like "considered judgments" or "second-order desires" or "reflectively endorsed preferences". The emphasis on these cognitively sophisticated attitudes is understandable, given that talk of 'values' generally accompanies talk of respect for patient autonomy, itself a highly intellectualized notion.

Yet there are many deep and stable attitudes towards persons, relationships, activities, objects, and pursuits that do not meet this cognitively sophisticated standard. Most IDD patients have loving and close relationships with family members and friends. ${ }^{3}$ They develop deep interests and passions for certain activities over others, and they will engage in patterns of behavior that reflect their interest and passion for the activity. They may have prolonged experience with particular forms of work or play that they develop a deep dislike for or aversion to. They may have abstract goals that they work towards achieving (such as independent living, social justice, or employment). These attitudes, while not always subject to explicit critical appraisal or reflective endorsement, form a way of seeing and navigating the world that is stable and distinctive. It is this orientation towards the world that we call an evaluative perspective.

Importantly, evaluative perspectives are not simply attenuated or diminished forms of values, although values can be part of the distinctive evaluative perspective for many people. 'Evaluative perspective' picks out a broader constellation of attitudes and habits of attention, ones

\footnotetext{
${ }^{3}$ We should note that the point here is not that love, care, and familial relations must form the basis of the trustee/patient relationship - although they may and frequently do. Rather, we point to the loving and close relationships that IDD patients enjoy with family and friends as material germane to discerning the patient's evaluative perspective, even if the patient lacks the abilities traditionally thought necessary for the articulation and application of that evaluative perspective in a decision-making context. Thank you to an anonymous reviewer for pointing this out.
} 
that individuals with full use of their cognitive capacities also have. Thus treating a patient's evaluative perspective as the object of moral concern is far more inclusive in which patients are counted as persons whose agency must be respected than traditional accounts.

To ensure that patients' actually elicited perspectives ground the collaboration central to supported decision-making, we propose that supported decision-making must be constrained by a commitment to non-domination. Non-domination is fundamentally concerned with ensuring that people are not vulnerable to outside interference that is divorced from their own evaluative perspectives (Pettit 1997; Pettit 2012). Domination occurs when a person is subject to the possibility that decisions or actions, over which they have (at least some) rightful say, will be redirected or frustrated by others unresponsive to that person's concerns, feelings, and values. However, unlike the independent control model, non-domination is less concerned with ensuring that people are free from interference simpliciter (Pettit 1997). Imposing constraints on a decisionmaking process in accord with considerations of non-domination does not necessarily require the minimization of involvement or "interference" from others.

A non-dominating supported decision-making process will focus on ensuring that the trustee lacks the power to commit the patient to decisions or actions in the absence of the right kind of relation to the patient's evaluative perspective. While it is important that the nondomination approach be compatible with ensuring that the decision is authentic to the patient, the importance of ensuring authenticity need not be justified on grounds of non-domination. On the non-domination model of supported decision-making, the goal is to reach a decision endorsed by the patient.

We submit that a decision is endorsed by the patient if it satisfies the following conditions:

[1] The decision can be publicly justified in terms of the patient's evaluative perspective; 
[2] The patient has been given opportunity to contest both the decision and the trustee's authority to engage in supported decision-making with patient;

[3] The patient cannot be interpreted as contesting

There are two further conditions, that are not required by endorsement per se, but do need to be in place for the deliberative process to secure the patient's capacity to endorse the decision at any given time:

[4] There are periodic opportunities for the patient to affirm that the trustee is an appropriate decision-maker.

[5] The patient's refusal to have a particular trustee serve as a decision-maker does not require justification on the patient's part.

The conditions for endorsement may seem fairly modest, as they focus primarily on the absence of contestation from the patient. However, this account of endorsement places significant constraints upon the decision-making process in order to create the conditions for its possibility. For it is imperative that the prospect of endorsement by the patient be guaranteed. A nondominating process strives not only to realize the goal of endorsement; it also strives to disallow the possibility that the process results in a decision not endorsed by the patient. Thus it is important to unpack in greater depth how considerations of non-domination constrain the decision-making process itself.

First, the non-domination approach requires that the patient's evaluative perspective be part of the foundation of the resulting decision. It is not enough that the decision merely not contradict the patient's evaluative perspective, or that the patient's evaluative perspective be the deciding factor in determining among decisions deemed appropriate without reference to the patient's evaluative perspective. Rather, the patient's evaluative perspective is part of the starting 
point for deliberation; it alone is capable of ruling out otherwise advisable or permissible courses of action. This requires that significant time and attention be dedicated to eliciting the patient's actual evaluative perspective; clarifying the patient's specific attitudes towards potential courses of action and how they relate to the group's current understanding of the patient's evaluative perspective might also be necessary.

Moreover, securing a foundational role for the patient's evaluative perspective requires that the trustee be able to defend any course of action in terms of considerations grounded in the patient's evaluative perspective. As Chew notes, "bad translations occur when the needs--the mode of signification--of the audience "blacks out" the original intent" (314). It is the responsibility of the trustee to ensure that the reasoning behind a decision is intelligible to others, but not in ways that undermine the decision's resonance with the patient themselves.

Second, the non-domination model emphasizes the importance of publicity within the collaboration. It is not enough that the trustee be able, if called upon, to justify the resulting decision in terms of the patient's evaluative perspective. Non-domination requires that patients actually have the opportunity to contest and to continue the process of deliberation. This implies that trustees must actually publicly defend the decision in considerations grounded in the patient's evaluative perspective. And just as the patient's evaluative perspective must be directly elicited, so too must the patient's possible contestation.

The emphasis on publicity is also reflected in the role for other participants in decisionmaking. Third parties (such as the medical provider, patient advocate, or a social worker) play an indispensable role in supported decision-making as safeguards for the patient against the possibility of being subject to decisions or actions divorced from the patient's evaluative perspective. Eliciting the patient's evaluative perspective is important in part because it gives third 
parties an opportunity to form their own judgments about the patient's evaluative perspective, even if there remains a pro tanto obligation to defer to the trustee's interpretation in cases of intractable disagreement between the trustee and a third party. Perceived discrepancy between the trustee's justification regarding a decision and the patient's elicited evaluative perspective should be challenged, and contestation by the patient should be prima facie supported by third parties. On the non-domination model, then, third parties can serve as an external check on the trustee's power, the process does not rely on the explicit contestation of the patient.

Finally, a non-domination approach to supported decision-making requires that the patient be given periodic opportunity to affirm that the trustee is the right decision-maker for the patient. Supported decision-making is not merely a series of disconnected instances of medical decisions, but a process that occurs against the background of a relationship between the patient and trustee. This background relationship is key to successful interpretation of the patient's evaluative perspective, but it also can create situations in which the trustee is unquestionably deferred to in their interpretation of the patient's evaluative perspective, regardless of whether they in fact are succeeding in their role as supporter. Moreover, this relationship may suffer from breakdowns in trust and comfort in ways that third parties are not privy to and cannot become privy to. Providing patients the opportunity periodically to affirm a specific individual as their trustee is essential to ensuring that the patient's evaluative perspective remains foundational to supported decisionmaking.

In addition to affording IDD patients periodic opportunity to affirm an individual as trustee, a non-domination approach to supported decision-making requires that any refusal of an individual as the patient's trustee must not require that the patient justify this refusal. Neurotypical patients need not provide clear reason for disqualifying specific individuals as decision-makers in the event 
that the patient becomes unable to serve as their own decision-maker. Given the increased risk of the evaluative perspective of IDD patients being disregarded or downplayed, it is all the more important that IDD patients not be required to justify their refusal to have a specific person serve as the patient's trustee. The commitment to treating the patient's evaluative perspective as foundational extends not just to the medical decision itself, but also to decisions about who supports the patient in decision-making.

A non-domination approach to supported decision-making emphasizes the creation of conditions for a decision-making process that hews closely to the patient's evaluative perspective. But it opens up the possibility for a fundamentally more shared form of medical decision-making, one which does not envision supported decision-making as an approximation of individual decision-making. But perhaps, in taking the 'shared' aspect of this sort of supported decisionmaking, it does not go far enough to ensure that IDD patients' rights are respected. In the remainder of this paper, we defend our approach to supported decision-making against a more aspirationally individualistic approach to supported decision-making - the mental prosthesis model.

\section{Why Not Mental Prosthesis?}

Silvers and Francis' advocacy for radical inclusion has led them to defend an approach to supported decision-making which aspires to the idea of mental prosthesis: "In the collaboration we envision, the trustee does not step into the subject's role in shaping a personalized notion of the good. Instead, as a prosthetic arm or leg executes some of the functions of a missing fleshly one without being confused with or supplanting the usual fleshly limb so, we propose, a trustee's reasoning and communicating can execute part or all of a subject's own thinking process without substituting the trustees own idea as if it were the subject's own." (Silvers and Francis, 2009, 485) This approach is one way to attempt to ensure that trustees like Chew are not displacing her son's 
voice with her own. Insofar as she sees herself as solely an appendage of her son's independent deliberation, she is not tempted to let her own evaluative perspective slip into her decision-making

Like our non-domination approach, the mental prosthesis approach of supported decisionmaking, requires the trustee to be primarily attuned to the evaluative perspective of the subject. However, their approach differs from ours in three key ways:

[1] The aim of supported decision-making on the mental prosthesis approach is to simulate individual decision-making, whereas the aim of the non-domination approach is to engage shared decision-making that is non-dominating of the patient's evaluative perspective.

[2] On the mental prosthesis approach, the trustee is instructed to aim at their own evaluative transparency rather than to be constrained by non-domination.

[3] On the mental prosthesis approach, the subject's perspective is understood as the "sole inspiration" for deliberation.

Let us look more closely at each of these three differences.

\section{Simulating Individual Decision-Making}

First, on Silvers and Francis' views, the goal of supported decision-making is to supplement the subject's mental capacities so as to approximate as close as possible to the individual decision-making process that the subject would have done if they did not require deliberative support. Silvers and Francis write that we must be careful to "distinguish between a process that remains a simulation of a particular human capacity that the subject lacks and a prosthetic process that compensates with a different capacity." (486)

Silvers and Francis use the example of competitive running and prosthetic racing feet to illustrate this difference. They defend the International Association of Athletics Federations ban on sprinters relying on assistive devices that roll on wheels because the point of a sprinting 
competition is to test a person's individual running abilities; it is not, more broadly, designed to test athlete's quickness in mobility from one location to another. Insofar as a person is competing in a sprinting competition, there will be appropriate limitations on the shape and function of the prosthetic mechanisms that a runner can use. Similarly, they argue, when it comes to supported decision-making, there should be appropriate limitations on the shape and function of the mental prosthesis that should be made available to the deliberating subject.

In contrast, our non-domination approach does not limit the deliberative supports that a trustee can offer solely to those processes that would simulate individual decision-making. We see three disanalogies between thinking about appropriate limits for prosthetic supports to sprinters and appropriate limits of deliberative supports for IDD patients.

First the criteria for appropriate prosthetic support for sprinting is based on one's notion of 'fair competition' but there is no reason to think that fair competition matters when it comes to supported decision-making. The reason why wheels are not deemed appropriate assistive technology is not that they do not allow people who use them to quickly get from one place to another, but rather the mode of movement on wheels is sufficiently different from running that it would not make sense to include athletes on wheels as engaged in the same competitive endeavor as runners -either on fleshly or on prosthetic feet. On the other hand, the choices made regarding health care or research participation do not close off opportunities for others in the same way. When it comes to exercising one's deliberative activities - such as voting or making medical decision-making - the standard of what counts as an appropriate deliberative support need not be sensitive to how closely the functioning mimics the functioning of such a role without the assistive technology. If a person who requires assistive technologies can make a better decision about how to vote (i.e. one that better reflects their evaluative perspective) with the aid of assistive thinking 
than one can without such aids, there is nothing yet unfair about participation with the use of these 'enhanced functions.' When it comes to supported decision-making, there is no zero sum game that may give certain people an unfair competitive edge.

The challenge for what counts as an "appropriate support" verses one of "trustee overstep" is not necessarily best determined by the level with which the deliberative process closely mimics individual decision-making. Which leads to the second disanalogy that we find: sprinting is judged on individual merit but deliberation [insofar as it is evaluated as successful or not] is often collaborative. Indeed Silvers and Francis, share this view of the collaborative nature of deliberation in general: "For all of us and not just for cognitively disabled people, conceptions of the good are socially scripted and interactively developed." (485) Why strip this collaborative aim for people with minimal verbal communication when it is appropriate for others? Rather than aim for simulating individual deliberation, we think that a more appropriate aim should be simulating shared decision-making that is non-dominating.

The final disanalogy is in thinking about a competitive sprinter's use of assistive technologies, we may be mislead to focus primarily on a 'lacked' capacity that is simulated rather than on supporting and amplifying present capacities. Insofar as the trustee is charged with limiting their interaction with the patient to only supplementing the capacities that the patient is lacking, the trustee must constantly be paying acute attention to what is lacking, rather than to engage in conversation and deliberation with the patient to ensure that their evaluative perspective is clarified and amplified - whatever means that perspective is communicated.

Taking these three disanalogies together, the point of mental prosthesis should not be limited in relation to whether it gives a person an advantage in their decision-making over others - at least not when it comes to how these assistive practices are going to be incorporated in medical 
decision-making. Second, thinking and deliberating is already a social endeavor. Why should supported decision-making prohibit the subject from engaging with a trustee that allows the process to amplify the social feature of the deliberative process rather than the independent control of the deliberative process? Given these disanalogies, we need not be limited to prosthetic mentality on the same model as prosthetic running blades. If the aim of supported decision-making is to ensure that the process respects and grows out of the evaluative perspective of the subject, mental prosthesis is one way to achieve such an aim, but it is not the only way and it may not be the most appropriate way in certain contexts or with certain persons.

\section{The Requirements of the Transparent Self}

Not only do Silvers and Francis envision a process that is completely controlled by the patient, they also envision the personality and evaluative perspective of the trustee to be diminished in their deliberative role:

The conceptualization should be stripped of the personality of the trustee. A fit trustee also will be someone whose identity is not invested in executing the trustee role, so as to be able to abstract himself from the process and the product. (Silvers and Francis 2009, 493)

But as we saw above, when thinking about the appropriate trustee relationship, we need not limit ourselves to the functionally similar deliberative procedure of a running blade when we have other models that can guide us. The running blade is a top-down form of control through prosthesis. It is the runner and the runner alone who controls what the prosthetic feet do. But there are other models of mentality and its relation to action that are more collaborative.

Consider for instance the way that an octopus explores and acts in its environment. Early on, biologists thought that the arms seemed "curiously divorced" from the brain, but recently studies have found much more coordination between the central brain and its arms (Smith 2016, 68). While the octopuses' brain retains a centralized authority, delegating and coordinating the movement of its limbs, the limbs still have leeway to explore the environment and report back 
findings. On this model, the octopuses' actions are still attributable to the individual animal, even if the intentionality is less centralized. Similarly, when it comes to supported decision-making, it may be fruitful to think about the possibilities of a more collaborative and coordinated model that the octopus makes vivid: allowing the arms to explore and employ some discretion in order to best attain the organism's overall goal.

Given the social nature of much deliberation, rather than think of oneself as an inanimate blade, guided solely by the intentional choices of the subject, a trustee may better understand their role as an exploratory or improvisorial arm, with some independent imaginative and discretionary powers, but always coordinated under the guiding authority of the subject.

This possibility is in line with the responsibilities attributed to trustees as Silvers and Francis envision them. They argue that trustees "must do more than weigh practicalities"- that is execute a plan of how to achieve the subject's conception of the good - "[t]hey must also transfer allegiances from conceptualizations that come to appear as chimerical to ones ready to be realized. Trustees charged with assisting people with cognitive disabilities in perfecting ideas of the good for them should be especially energetic in presenting and exploring alternatives through a revisionary process. Simply rejecting an idea does not do the job." (491) We agree with these robust requirements of the trustee. Being the trustee requires discretion, resourcefulness and the use of one's own imaginative capacities and judgments. For this reason, instructing the trustee to aim at becoming transparent and to not be invested in their role as trustee, may constrain a more proactive orientation to the shared deliberation.

One of the agential capacities that people have is to trust specific others to support them in their decision-making. When we trust others, we are not merely relying on their abstract cognitive capacities. We often trust their judgment, which may be hard won given their distinctive 
experiences and histories, and we often trust that they care about us and are invested in our life projects and commitments. It is unclear whether all IDD patients would want their trustees to abstract away from all the features that distinctively make them trustworthy to the patient in the first place. The patient's evaluative perspective need not be limited to the outcomes of the decision; patients may also have views and preferences about the shared decision-making process itself. While some patients may prefer an abstracted trustee, others may wish to have the support and collaboration of a friend or loved one, with deep personal investment in the relationship. Our nondomination approach does not weigh in on which of these wishes is better. What matters is that surrogates approach their role in a manner endorsed by the patients. This opens up different styles of shared decision-making between patient and trustee, depending on the expressed evaluative perspective of the patient and the judgments of the trustee about how to best inhabit their supportive role.

\section{The Patient as the Sole Inspiration}

We agree that the patient perspective should have an authoritative and active role to play in deliberation, but we are not sure that this requires that the patient's perspective be the sole inspiration for the deliberative process for many of the reasons we have already articulated. The mental prosthesis approach differs from the non-domination approach in its view of the purpose of collaboration. While the mental prosthesis approach envisions the patient and trustee working together to simulate an individual reasoning and decision-making process, the non-domination approach envisions the patient and trustee functioning as independent parts of a shared reasoning and decision-making process, one constrained by the patient's evaluative perspective.

In view of this, one might wonder whether what we are articulating in this paper is a genuine alternative account of supported decision-making to the mental prosthesis model, or rather 
another approach intended for a different patient population. Specifically, perhaps we should see the non-domination model as an approach to supported decision-making suitable for those patients who require greater support in their decision-making due to the dormancy or absence of capacities assumed necessary to independently articulate an evaluative perspective that can establish the foundation for decision-making. In contrast, the mental prosthesis approach may be thought to be better suited for IDD patients able to independently advocate for and articulate their own evaluative perspective. $^{4}$

We think our model is genuinely an alternative approach for all IDD patients. All IDD patients, including those with who can independently advocate for their own evaluative perspective, may prefer a more proactive partner in the trustee. We believe that the social and collaborative nature afforded by the non-domination model is appropriate quite broadly. But we do concede that the differences between the two models pose a potential problem for the nondomination model. They highlight that the non-domination model places less emphasis on the significance of independence of the patient in the decision-making process.

Given the aims of supported decision-making to secure respect for patients who historically have been deprived of control over their medical decisions, one may argue that a non-domination approach does not go far enough in actually supporting the patient and her distinctive evaluative perspective. One might object that the non-domination approach does not articulate a form of supported decision-making at all. Specifically, one might object that the non-domination approach is not meaningfully different from a substituted judgment approach to decision-making. If this were so, then we would run the risk of articulating a form of supported decision-making that only

\footnotetext{
${ }^{4}$ Thank you to an anonymous reviewer for suggesting this way of thinking about the relationship between the nondomination model and the mental prosthesis model and encouraging us to be clearer about why we do not view ourselves as merely articulating a separate approach to supported decision-making for a different patient population.
} 
superficially protected the evaluative perspectives of IDD patients — especially those patients who may be unable to communicate on their own behalf in ways that are recognized by the majority of individuals. We recognize this as an important challenge to our view. In the final section of the paper, we will articulate some reasons why we think that the non-domination model is importantly different from substituted judgment.

\section{Is the Patient Supported Enough?}

The non-domination approach anticipates that the trustee will be responsible for articulating the patient's evaluative perspective, but also that the trustee will engage in some independent forms of deliberative activity. Given the independence of the trustee as a deliberative participant, one might worry that non-domination hews far closer to substituted judgment than to the vision of supported decision-making.

However, this objection overlooks some key distinctions. First, the substituted judgment approach allows the trustee to merely imagine the patient's evaluative perspective. While substituted judgment presumes the trustee has some general knowledge of the patient's evaluative perspective, the idea behind substituted judgment is that the trustee uses this knowledge to develop a picture of how the patient's evaluative perspective, appropriately informed and idealized, would extend to the particular decision-making context. But there is no requirement, or even expectation, that the patient's actual perspective on this decision will factor into deliberation. By contrast, the non-domination approach requires that the trustee and other participants directly and publicly elicit the patient's evaluative perspective. Moreover, this actually elicited evaluative perspective is indispensable to the decision-making process. Thus the non-domination approach insists that deliberation be responsive to the actual patient, not to an imagined form of the patient. 
Second, trustees on the substituted judgment approach assume decision-making authority for the patient. This decision-making authority is awarded to the trustee on an assumption that they have specific insight into the patient's evaluative perspective, insight that can guide decisionmaking on behalf of the patient. Thus there is no expectation, on the substituted judgment model, that the trustee offer a public justification of their interpretation of the patient's evaluative perspective. It is simply assumed that the trustee's interpretation of the patient's evaluative perspective is authoritative. This differs from the non-domination approach, in which the trustee is not authoritative with respect to the patient's evaluative perspective. The trustee has a responsibility to the patient and to the medical team to actually defend their interpretation of the patient's evaluative perspective and the resulting decision.

Third, the substituted judgment approach is an individualistic approach to medical decision-making. The trustee is tasked with making decisions both constrained by the patient's evaluative perspective and informed by the current clinical circumstances. Thus acquiring key clinical information will require the input of the health care provider. Moreover, the trustee may solicit advice or feedback from other parties should they feel it useful or helpful. But ultimately, these are decisions for the trustee to make. On the substituted judgment account, contestation from third parties of the decision is appropriate only if the trustee is operating outside the bounds of their authority, or if there is reason to reevaluate whether the individual is suitable to serve as trustee.

In contrast, the non-domination approach is fundamentally a collaborative approach to medical decision-making, constrained by the evaluative perspective of the patient. This commitment to collaboration necessitates the creation of opportunities for contestation; a collaboration that recognizes contestation only when third parties take it upon themselves to 
challenge the outcome of a deliberative process--and this challenge has been deemed an admissible challenge to the trustee's authority--seems far from the spirit of collaborative decision-making. Thus the non-domination approach, unlike substituted judgment, does not award the trustee sole authority over the decision.

In sum, the non-domination approach, both in its commitment to ensuring that the trustee is responsive to the actually elicited evaluative perspective of the patient and its emphasis on the importance of public deliberation and opportunities for contestation, does not award the trustee the kind of power over decision-making that the substituted judgment approach allows for. Thus the non-domination approach, while giving the trustee greater leeway in deliberation and decisionmaking than the mental prosthesis approach, protects the patient from having their evaluative perspective overlooked or overridden in a way that the substituted judgment approach does not.

The outlined approach above raises a number of practical challenges that must be thoughtfully addressed, ranging from concerns about identifying those communicative techniques that accurately elicit the patient's evaluative perspective to worries about the degree of burdensomeness for the trustee, which may incentivize hasty or unjustified judgments about the patient's evaluative perspective. ${ }^{5}$ While it goes beyond the scope of this paper to address these challenges - challenges that confront not just any proponent of supported decision-making, but that regularly crop up in thinking about medical decision-making on behalf of neurotypical patients ${ }^{6}$ - we recognize the need to tackle these questions in thinking about supported decision-

\footnotetext{
${ }^{5}$ Thank you to two anonymous reviewers for raising these as reasonable concerns about the prospect of achieving the outlined approach in the paper.

${ }^{6}$ It is not uncommon in the clinical context, for example, for there to be regular and extensive goals of care conversations with surrogate decision-makers where there is disagreement between the team and the family about the appropriate course of action. And it is not uncommon for clinical ethicists to advise in such circumstances that medical professionals attend to the potential burdensomeness of such repeated conversations and to recommend strategies for mitigating those burdens and optimizing conversations with families.
} 
making in order to ensure that we have not outlined a process that potentially undercuts the extent to which the patient's evaluative perspective guides decision-making.

But one might object to the degree of leeway that has been afforded to the trustee in even the conceptual formation of our approach to supported decision-making. The aim of supported decision-making is to facilitate authentic decisions for patients who may only ever be spoken on behalf of. By allowing greater room for the trustee's own perspective, it may be argued that the non-domination approach undermines the very intent of supported decision-making. Therefore, the individualism at the heart of the mental prosthesis approach is a desirable feature; it affords the patient's evaluative perspective the respect it deserves.

We grant that our approach allows for the trustee's evaluative perspective to have greater potential influence over the resulting decision. But we are comfortable with this outcome. We believe that the non-domination approach is closer to the way most people actually make and actually prefer to make decisions. And this includes patients who have no need for supported decision-making. While it is common to articulate an ideal of independent medical decisionmaking for patients in full use of their cognitive capacities, it is far more common for patients to base their decisions on the recommendations and perspectives of others involved in the deliberation (Ubel, Scherr, and Fagerlin 2017). This may include family members, friends, health care providers, or even strangers on internet forums. As is well noted by advocates of supported decision-making, most people--regardless of the presence or absence of an intellectual or developmental disability--want to be supported in making their medical decisions. There are perhaps some occasions, such as labor and delivery plans or the choice to undergo prophylactic double mastectomy surgery, where the ability to independently make medical decisions is of paramount importance since these decisions are necessary for our ability to lead our lives according 
to our values. But often, we make medical decisions just to get healthy or to alleviate pain. Medical decisions may not be expressions of our deep values, they are often just instrumental so that we can go on to make the truly meaningful life choices elsewhere. This doesn't mean that patients should be subject to the domination of others while making such decisions, but aiming at maximal autonomy is only one way to safeguard against domination.

It may be pointed out that the non-domination approach still treats IDD patients, and the moral status of their evaluative perspective, differently, in that it articulates a different ideal of medical decision-making. Even if it is true that patients in full use of their cognitive capacities often make decisions in a more collaborative way, it is still open to them to make decisions informed only by their own evaluative perspective. Patients with full use of their cognitive capacities have the authority to exclude certain contributions or considerations, and to make medical decisions largely independently. The mental prosthesis approach, in its encouraged view of the trustee as a mere appendage of the patient, protects this authority. By contrast, the nondomination approach does not award such authority to IDD patients; it only ensures the predominance of these patients' evaluative perspectives in the resulting decisions. This is the relevant sense in which the non-domination approach may be understood as failing to afford the patient's evaluative perspective due respect.

We recognize the noted asymmetry between the non-domination approach for supported decision-making and the autonomy-centered ideal common for patients in full use of their cognitive capacities. Perhaps maintaining this asymmetry would reflect an unacceptable difference in the respect we give to the evaluative perspectives of IDD patients. However, we think that this asymmetry should be resolved by reconsidering our ideal for patients in full use of their cognitive capacities. In other words, we are skeptical that this ideal of full independence is a more 
appropriate aim for patients than making decisions that reflect their values while being free from domination in the process. To defend this point goes beyond the scope of this paper. However, we reject the claim that we should resolve the asymmetry by defending an approach to supported decision-making that strives to approximate a wholly independent decision-making process. Rather, we think this should be cast as an invitation to reflect on whether the non-domination approach may be more appropriate for all patients.

\section{Works Cited}

Buchanan, Allen E and Dan W. Brock. 1990. Deciding for others: the ethics of surrogate decision-making. Cambridge: Cambridge University Press.

Chew, Kristina. 2013. Autism and the task of the translator. In Worlds of autism: Across the spectrum of neurological difference, eds. Joyce Davidson and Michael Orsini, editors, 30517. Minneapolis: University of Minnesota Press.

Doe ex. rel. Tarlow v. District of Columbia, 489 F.3d 376 (D.C. Cir. 2007)

Godfrey-Smith, Peter. 2016. Other minds: the octopus, the sea, and the deep origins of consciousness. New York: Farrar, Strauss, and Giroux.

Howard, Dana and David Wendler. 2020. Beyond instrumental value: Respecting the will of others and deciding on their behalf. In The Oxford handbook of philosophy and disability, eds. Adam Cureton and David Wasserman. Oxford: Oxford University Press

Pettit, Philip. 1997. Republicanism: A theory of freedom and government. Oxford: Clarendon.

Pettit, Philip. 2012. On the people's terms: A republican theory and model of democracy. Cambridge: Cambridge University Press.

Silvers, Anita and Leslie Pickering Francis. 2009. Thinking about the good: Reconfiguring liberal metaphysics (or not) for people with cognitive disabilities. Metaphilosophy 40 (3-4): 47598.

Ubel, Peter A., Karen A. Scherr, and Angela Fagerlin. 2017. Empowerment failure: how shortcomings in physician communication unwittingly undermine patient autonomy. American Journal of Bioethics 17(11): 31-9. 
